\title{
The cerebral network of COVID-19-related encephalopathy: a longitudinal voxel-based 18F-FDG-PET study
}

\author{
Aurélie Kas $^{1}$ (D) $\cdot$ Marine Soret ${ }^{1} \cdot$ Nadya Pyatigoskaya $^{2} \cdot$ Marie-Odile Habert $^{1} \cdot$ Adèle Hesters $^{3} \cdot$ Loic Le Guennec $^{3}$. \\ Olivier Paccoud ${ }^{4}$. Stéphanie Bombois ${ }^{5}$. Cécile Delorme ${ }^{3}$. on the behalf of CoCo-Neurosciences study group and \\ COVID SMIT PSL study group ${ }^{1}$
}

Received: 24 September 2020 / Accepted: 22 December 2020 / Published online: 15 January 2021

(C) The Author(s), under exclusive licence to Springer-Verlag GmbH, DE part of Springer Nature 2021, corrected publication 2022

\begin{abstract}
Purpose Little is known about the neuronal substrates of neuropsychiatric symptoms associated with COVID-19 and their evolution during the course of the disease. We aimed at describing the longitudinal brain metabolic pattern in COVID-19related encephalopathy using 18 F-FDG-PET/CT.

Methods Seven patients with variable clinical presentations of COVID-19-related encephalopathy were explored thrice with brain 18F-FDG-PET/CT, once in the acute phase, 1 month later and 6 months after COVID-19 onset. PET images were analysed with voxel-wise and regions-of-interest approaches in comparison with 32 healthy controls.

Results Patients' neurological manifestations during acute encephalopathy were heterogeneous. However, all of them presented with predominant cognitive and behavioural frontal disorders. SARS-CoV-2 RT-PCR in the CSF was negative for all patients. MRI revealed no specific abnormalities for most of the subjects. All patients had a consistent pattern of hypometabolism in a widespread cerebral network including the frontal cortex, anterior cingulate, insula and caudate nucleus. Six months after COVID-19 onset, the majority of patients clinically had improved but cognitive and emotional disorders of varying severity remained with attention/executive disabilities and anxio-depressive symptoms, and lasting prefrontal, insular and subcortical 18F-FDG-PET/CT abnormalities.

Conclusion The implication of this widespread network could be the neural substrate of clinical features observed in patients with COVID-19, such as frontal lobe syndrome, emotional disturbances and deregulation of respiratory failure perception. This study suggests that this network remains mildly to severely impaired 6 months after disease onset.
\end{abstract}

Keywords Glucose metabolism · 18F-FDG-PET $\cdot$ SARS-CoV-2 $\cdot$ Prefrontal impairment $\cdot$ COVID-19

Stéphanie Bombois and Cécile Delorme contributed equally to this work.

This article is part of the Topical Collection on Neurology

Aurélie Kas

aurelie.kas@aphp.fr

1 Sorbonne Université, AP-HP, Hôpitaux Universitaires Pitié-Salpêtrière Charles Foix, Service de Médecine Nucléaire and LIB, INSERM U1146, 75013 Paris, France

2 Département de Neuroradiologie, Sorbonne Université, AP-HP, Hôpitaux Universitaires Pitié-Salpêtrière Charles Foix, 75013 Paris, France
3 Département de Neurologie, Sorbonne Université, AP-HP, Hôpitaux Universitaires Pitié-Salpêtrière Charles Foix, 75013 Paris, France

4 Service de Maladies infectieuses et Tropicales, Sorbonne Université, AP-HP, Hôpitaux Universitaires Pitié-Salpêtrière Charles Foix, 75013 Paris, France

5 Sorbonne Université, AP-HP, Hôpitaux Universitaires Pitié-Salpêtrière Charles Foix, Institut de la Mémoire et de la Maladie d'Alzheimer, 75013 Paris, France 


\section{Introduction}

Since December 2019, severe acute respiratory syndrome coronavirus 2 (SARS-CoV-2) is causing a worldwide outbreak with substantial morbidity and mortality. Neuropsychiatric complications of COVID-19 including encephalopathy, encephalitis and anxio-depressive disorders have been increasingly described [1], but little is known about their neuronal substrates and pathogenic mechanisms. These manifestations could be caused by direct effects of the virus on the nervous system, para-infectious or post-infectious immune-mediated disease, or neurological complications of the systemic effects of COVID-19 and critical illness, these hypotheses being not mutually exclusive. Several specific patterns in structural magnetic resonance imaging (MRI) have been described but it remains often non-contributive [2] and functional imaging data is still limited. In line with published cases [3, 4], a previous case series of four patients suggested the involvement of the prefrontal cortex and cerebellum in acute COVID-19related encephalopathy using $18 \mathrm{~F}$-fluorodeoxyglucose (18FFDG) PET/CT analysed visually [5]. However, the interpretation of these results was limited due to the qualitative nature of the data. Here, we report a series of seven patients with acute encephalopathy in the context of COVID-19, who were investigated during the acute phase and longitudinally up to 6 months after COVID-19 onset with 18F-FDG-PET/CT analysed quantitatively in comparison with healthy controls using regions-of-interest and voxel-based approaches.

\section{Methods}

\section{Patients}

Seven consecutive cases of COVID-19-related encephalopathy (median age $63 \mathrm{y} / \mathrm{o}$, range 50-72 y/o; 4 men) hospitalized in Neurology or Psychiatry wards were explored with $18 \mathrm{~F}$ FDG-PET/CT as part of their clinical investigation, from March to June 2020 in the Pitié-Salpêtrière Hospital in Paris, France.

The diagnosis of COVID-19-related encephalopathy was based on new-onset cognitive impairment with central focal neurological signs or seizures in the context of COVID-19, in the absence of another cause of encephalopathy. The diagnosis of COVID-19 was confirmed in all patients by a positive reverse-transcriptase polymerase chain reaction (RT-PCR) assay from a nasopharyngeal swab sample. All patients underwent cerebrospinal fluid (CSF) analysis, electroencephalogram (EEG), brain MRI and brain 18F-FDG-PET/CT. Other infectious and autoimmune disorders were excluded by extensive diagnostic workup. The evolution of their neuropsychiatric symptoms, including cognitive, behavioural and emotional status was assessed within the first 2 months, and 3 to 6 months after COVID-19 onset by a multidisciplinary team in our memory clinic. A comprehensive battery of validated cognitive tests was used to test global cognitive performances, working memory, executive functions, attention, verbal and visual episodic memory, praxis, visuo-constructive abilities and social cognition. The speech therapist tests battery assessed the global language abilities, and more specifically fluencies, denomination and semantic abilities. Emotional aspects were assessed by an interview conducted by a psychologist.

Patients received information and agreed to the use of their medical data in accordance with French regulations. The study was approved by the Sorbonne University Ethics Committee (CER-202028 on 24/04/2020).

\section{Brain PET imaging}

PET imaging was performed at the acute phase, 1 month later and 6 months after COVID-19 onset, following the same protocol: a 10-min acquisition was performed with a hybrid PET/ CT system (Biograph mCT Flow, Siemens Healthcare) $30 \mathrm{~min}$ after the injection of $18 \mathrm{~F}-\mathrm{FDG}(2 \mathrm{MBq} / \mathrm{kg})$. Images were reconstructed using an iterative algorithm and corrected for attenuation and scatter.

PET images were spatially normalized into the Montreal Neurological Institute (MNI) space with SPM8 (https://www.fil. ion.ucl.ac.uk/spm/), smoothed and normalized in intensity using the pons as reference region. Two-sample $t$ tests were used to identify glucose metabolism changes in the whole patient group and in each patient individually in comparison with 32 healthy subjects (age $56.3 \pm 8.7 \mathrm{y} / \mathrm{o}$; range $40-72 \mathrm{y} / \mathrm{o} ; 19 \mathrm{men}$ ). The same $\mathrm{PET} / \mathrm{CT}$ system and imaging protocol were used for patients and controls. Age was set as a confounding variable. SPM(T) maps were thresholded with the primary defining threshold set at $p<0$. 05 at the peak level, corrected with the family-wise error rate (FWE) method except for one contrast (patients $>$ controls). For the latter analysis, the threshold was decreased at $p<0.001$ uncorrected at the peak level. A region-of-interest (ROI) analysis was performed with Scenium software (Siemens Healthcare).

\section{Results}

\section{Clinical and imaging features during acute encephalopathy}

Patients' clinical presentation, main biological and imaging features are given in Table 1. A detailed description of four of the patients is available in a previous paper [5]. The patients had varied COVID-19 severity: three patients required mechanical ventilation $(\# 1,2,7)$ and three required nasal oxygen (\#4, 5, 6). Patient \#3 was hospitalized but did not need oxygen. They had wide-ranging neurological presentations and 


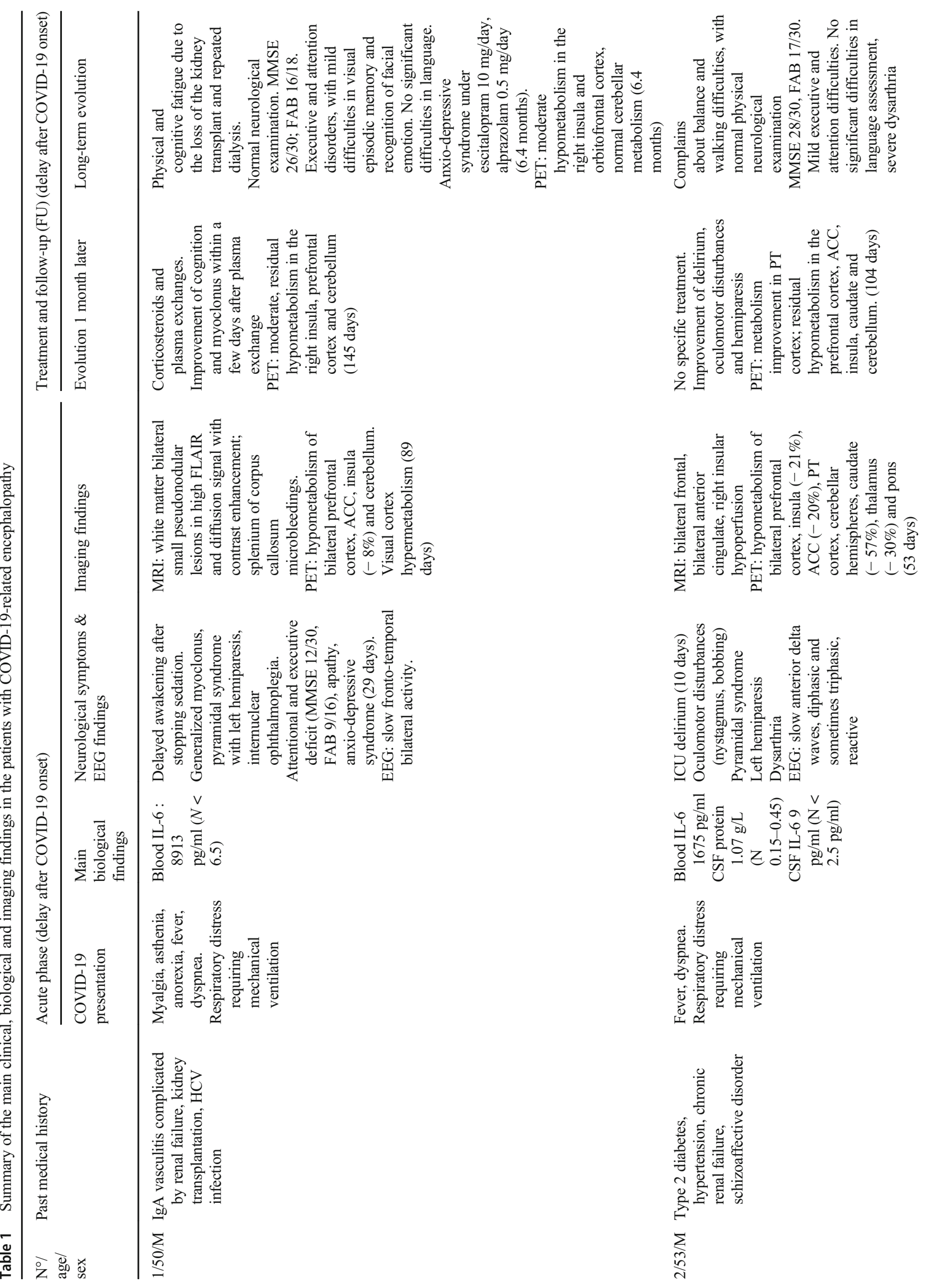




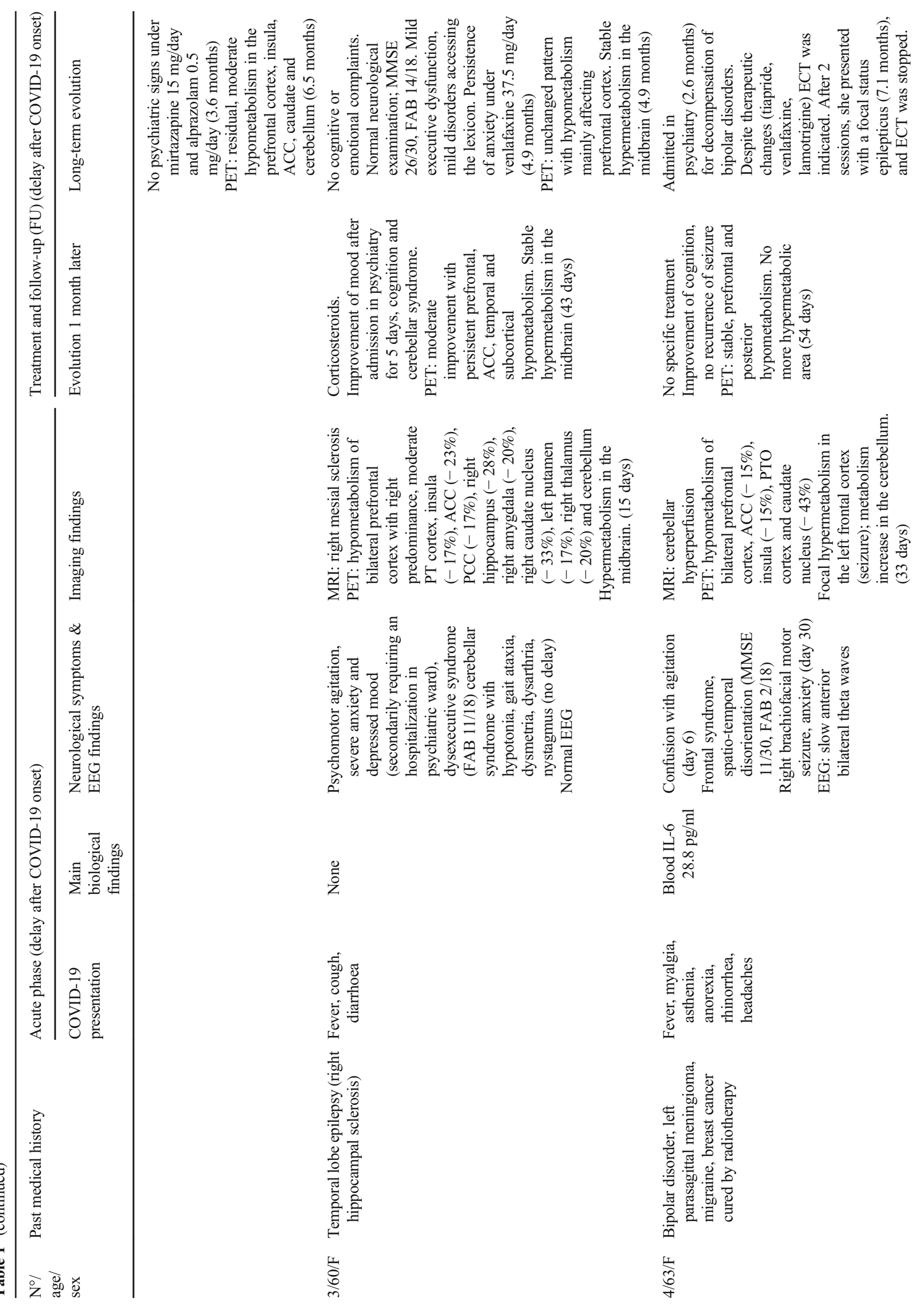




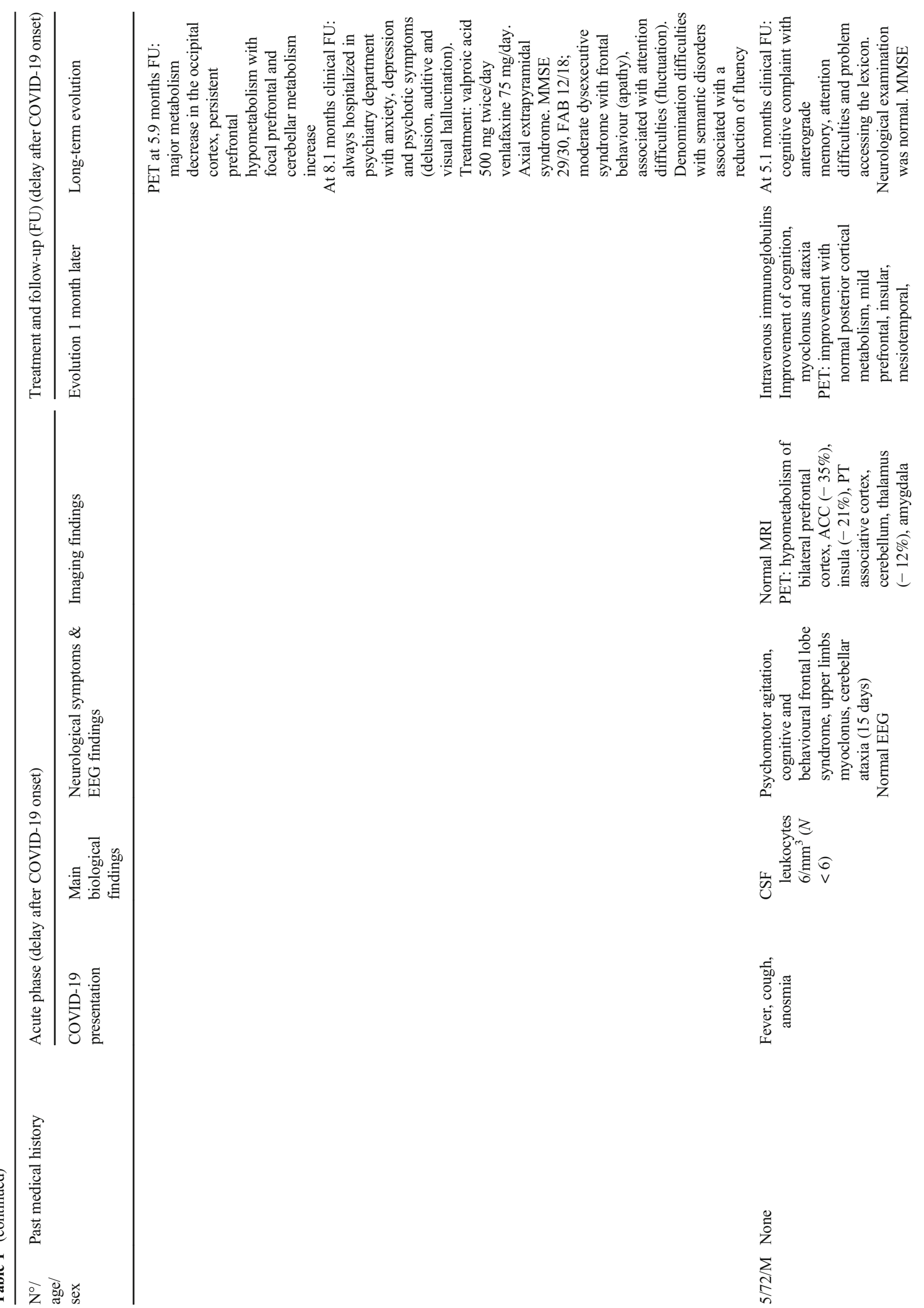




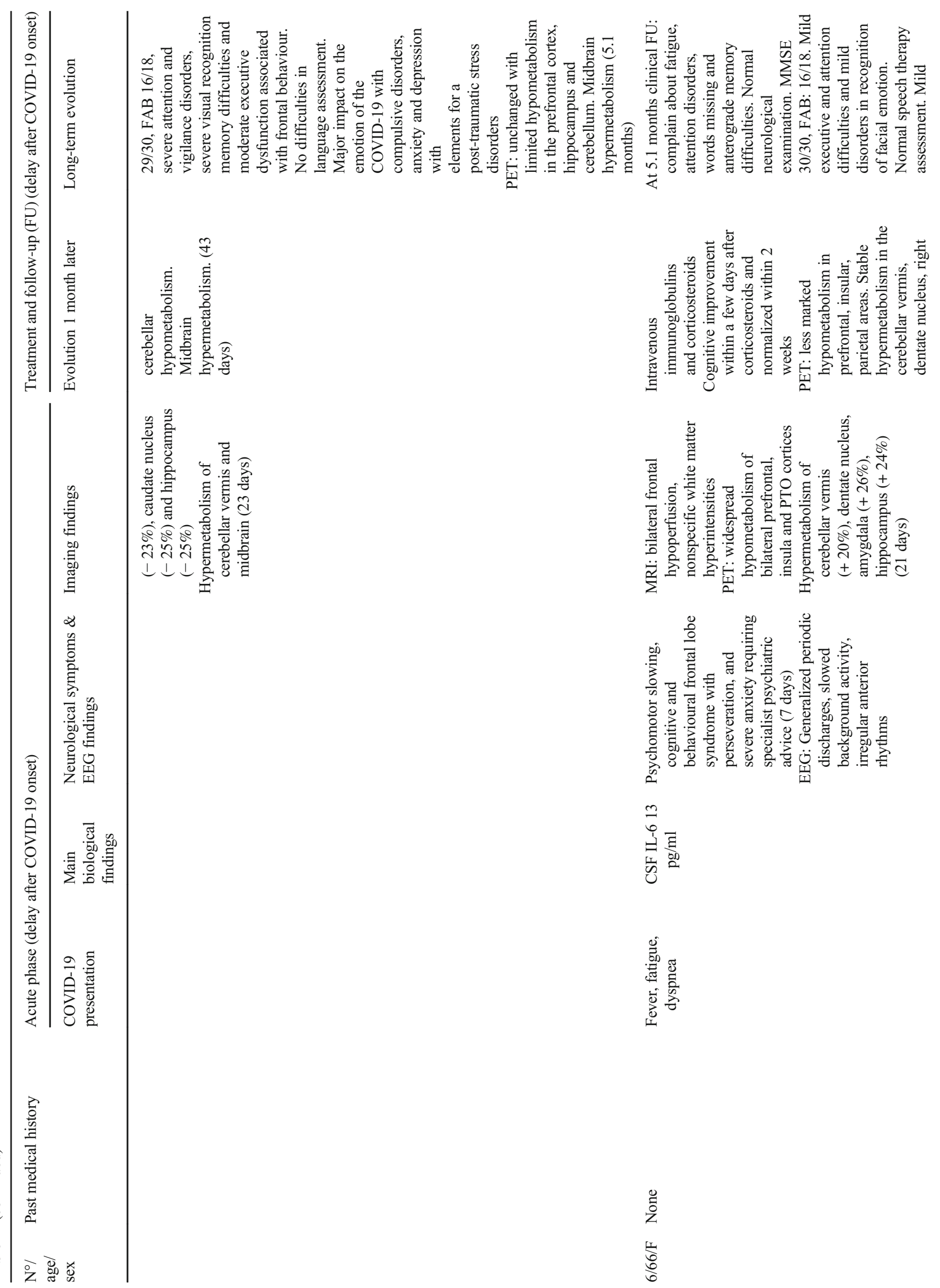




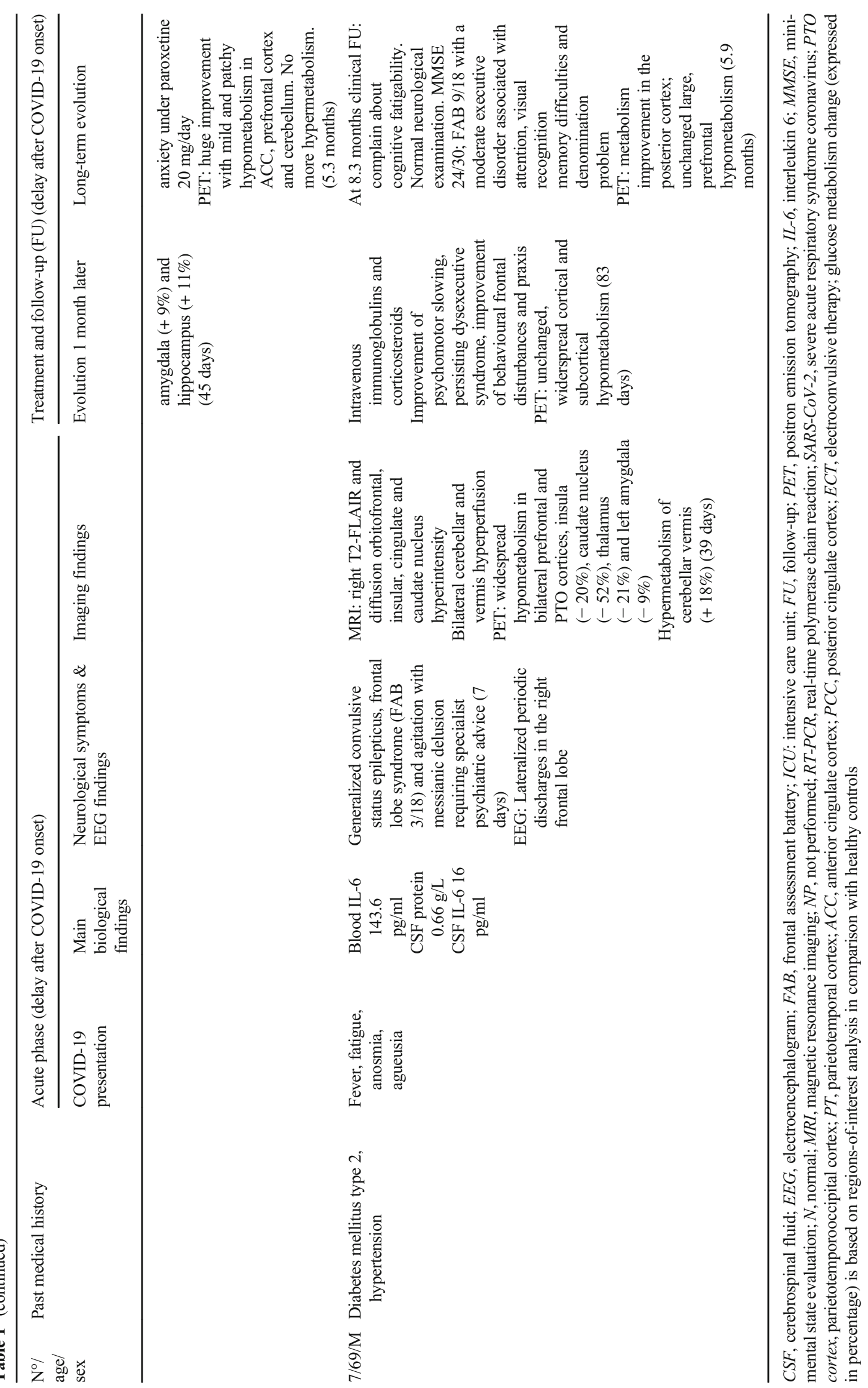


variable degrees of cognitive dysfunction. They all experienced severe executive deficit and frontal behaviour changes with apathy. A cerebellar syndrome was observed in two patients (\#3, 5), myoclonus in two (\#1, 5), psychiatric manifestations in five (\#1, 3, 4, 6, 7) and oculomotor disturbances in two (\#1, 2). One patient had status epilepticus (\#7) and one patient had a focal motor seizure (\#4). One patient had delayed awakening (\#1) and one patient had delirium (\#2) following sedation stopping in the intensive care unit. Blood IL-6 levels were increased in 5/5 patients and CSF IL-6 levels in 3/4 patients. One patient had CSF leukocytes $>5 \mathrm{~mm}^{3}(\# 5)$, and two patient had CSF protein $>0.4 \mathrm{~g} / \mathrm{L}(\# 2,7)$. SARS-CoV-2 RT-PCR in the CSF was negative in all patients.

The median delay between the onset of COVID-19, neurological symptoms and 18F-FDG-PET/CT was 7 days (range 0-29) and 33 days (range 15-89), respectively.

In the whole group of patients vs. controls, voxel- and ROIbased analyses showed a prominent hypometabolism of the prefrontal cortex prevailing on the right side, bilateral insula (14\% decrease vs. controls), anterior cingulate $(-20 \%)$, right $(-33 \%)$ and left $(-26 \%)$ caudate nucleus $(p<0.05$ corrected, Fig. 1, Tables 2 and 3). The SPM-based analysis showed mild hypermetabolism in the vermis, dentate nucleus and pons $(p<0.05$, Table 2$)$.
Individual analyses confirmed a severe, bilateral prefrontal hypometabolism in 7/7 patients, including the rectus gyrus ( $p$ $<0.05$ FWE corrected, Fig. 2, Table 4). In every patient, hypometabolism expanded bilaterally to the anterior cingulate and insula ( $p<0.05$ FWE corrected, Table 4$)$. A mild to moderate parietotemporal associative hypometabolism was observed in $4 / 7$ patients, whereas two others had a severe, spread parietotemporooccipital hypometabolism (\#6, 7). Hypermetabolism was found in the visual cortex in patient \#1 due to visual stimulation after 18F-FDG injection and in the left frontal cortex in patient \#4 due to focal seizure. Notably, bilateral mesiotemporal hypermetabolism was found in one patient (\#6) without T2-MRI hyperintensity, whereas hypometabolism was observed in patient \#3 with known hippocampal sclerosis and in patient \#5 having hippocampal atrophy. Metabolism was decreased in the caudate nucleus in five patients $(\# 2-5,7)$ and thalamus in two $(\# 2,3)(p<0.05$ corrected). Metabolism was decreased in the bilateral cerebellar hemispheres in all patients except one (\#4) but increased in the vermis in $5 / 7$ patients $(p<0.05)$.

Structural MRI showed no cerebrovascular disease or specific COVID-related abnormalities except for one patient \#1 presented with typical white matter enhanced lesions, as previously described [2]. Metabolism changes were not

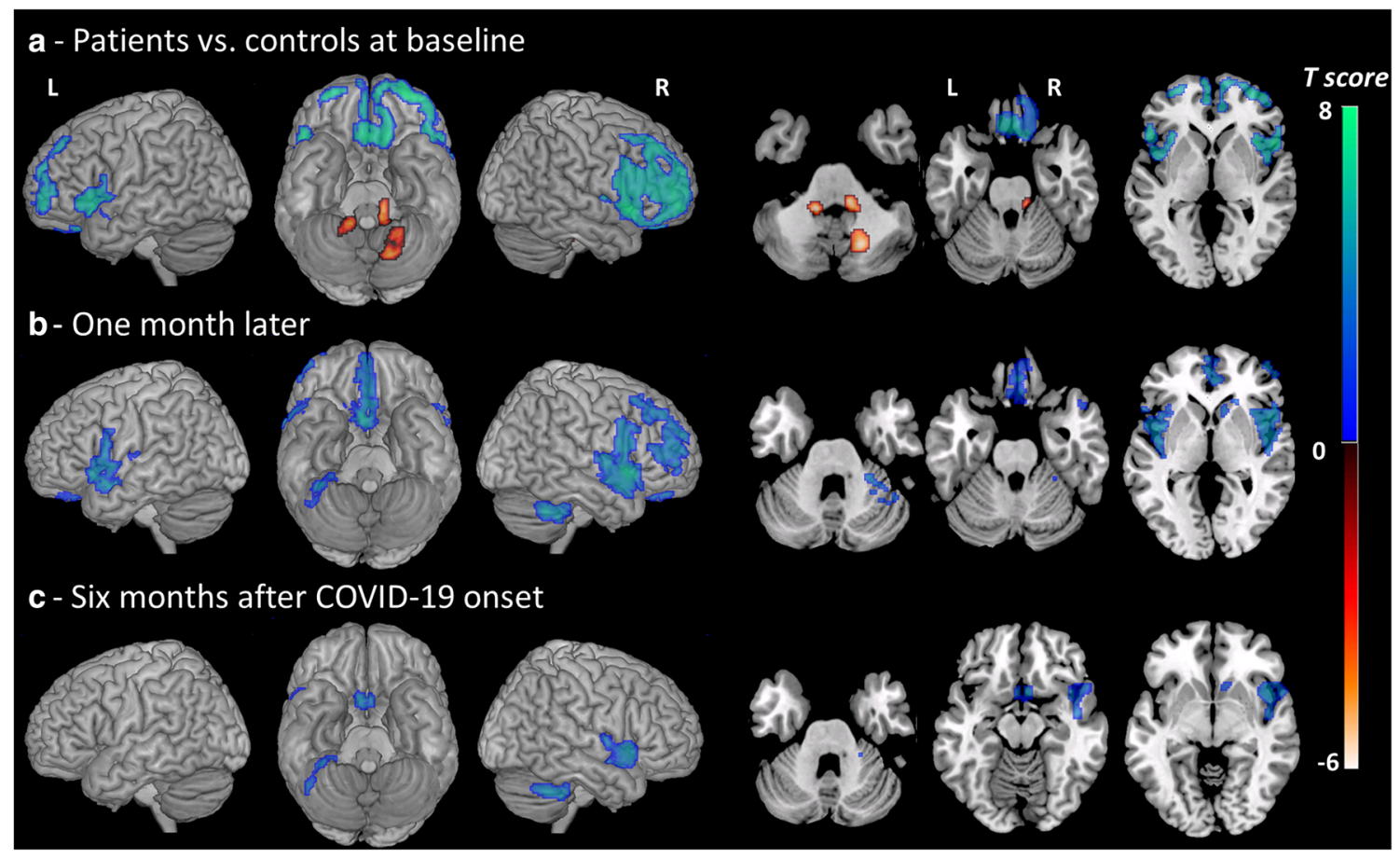

Fig. 1 Brain metabolism changes in the whole group of patients $(n=7)$ vs. controls $(n=32)$ in the acute phase, 1 month later and 6 months after COVID-19 onset. Hot and cool color scales show regions with hypermetabolism and hypometabolism in patients vs. controls, respectively. The SPM maps are projected onto a surface rendering and onto axial views of the customized MRI template. The axial slices are shown using neurological conventions (right is right). $\mathrm{R}$, right; $\mathrm{L}$, left. At baseline, hypometabolism was found in the bilateral prefrontal cortex with right predominance, insula, anterior cingulate and caudate $(p<$ 0.05 corrected). The analysis showed a mild hypermetabolism in the vermis, dentate nucleus and pons $(p<0.05)$. One month later, hypometabolism was limited to the mediofrontal, right dorsolateral areas, olfactory/rectus gyrus, bilateral insula, right caudate nucleus and cerebellum $(p<0.001)$. Six months after COVID-19 onset, metabolism decrease was observed in the same regions but they were less extended ( $p$ $<0.001)$ 
Table 2 18F-FDG-PET findings in the whole group vs. 32 healthy controls with the SPM approach, at the acute phase, 1 month later and 6 months after COVID-19 onset

\begin{tabular}{|c|c|c|c|c|c|c|c|}
\hline \multirow[t]{2}{*}{ Location (BA) } & \multicolumn{3}{|c|}{ Coordinates MNI (mm) } & \multirow{2}{*}{$\begin{array}{l}\text { Cluster size } \\
\mathrm{k}_{\mathrm{E}}\end{array}$} & \multicolumn{3}{|c|}{ Voxel level } \\
\hline & $x$ & $y$ & $z$ & & $P$ corr & P uncorr & $T$ score \\
\hline \multicolumn{8}{|l|}{ Baseline } \\
\hline \multicolumn{8}{|l|}{ Patients $(n=7)<$ controls } \\
\hline Right insula (BA47) & 48 & 16 & -4 & 3832 & $<0.001$ & $<0.001$ & 8.8 \\
\hline Left frontal medial gyrus (BA10) & -4 & 56 & 8 & 939 & $<0.001$ & $<0.001$ & 8.3 \\
\hline Left insula (BA47) & -34 & 22 & -6 & 566 & $<0.001$ & $<0.001$ & 8.2 \\
\hline Right rectus gyrus (BA11) & 11 & 24 & -26 & 303 & $<0.001$ & $<0.001$ & 7.1 \\
\hline Right middle temporal gyrus (BA21) & 68 & -16 & -18 & 135 & $<0.001$ & $<0.001$ & 6.8 \\
\hline Right middle cingulate (BA32) & -4 & 30 & 34 & 107 & $<0.001$ & $<0.001$ & 6.5 \\
\hline \multicolumn{8}{|l|}{ Patients $(n=7)>$ controls } \\
\hline Right pons* & 12 & -32 & -34 & 138 & $>0.05$ & 0.002 & 3.0 \\
\hline Left pons* & -14 & -42 & -42 & 65 & $>0.05$ & 0.003 & 2.9 \\
\hline \multicolumn{8}{|l|}{ Early assessment } \\
\hline \multicolumn{8}{|l|}{ Patients $(n=7)<$ controls } \\
\hline Right insula (BA47) & 48 & 17 & -4 & 1883 & $<0.001$ & $<0.001$ & 7.1 \\
\hline Left temporal sup gyrus (BA38) & -40 & 11 & -16 & 964 & 0.01 & $<0.001$ & 6.6 \\
\hline Right olfactory gyrus (BA25) & 4 & 15 & -16 & 2430 & 0.002 & $<0.001$ & 6.2 \\
\hline Right cerebellum & 40 & -50 & -46 & 304 & $<0.001$ & 0.002 & 5.9 \\
\hline Right middle frontal gyrus (BA10) & 46 & 52 & 0 & 408 & 0.005 & $<0.001$ & 4.9 \\
\hline \multicolumn{8}{|l|}{ Patients $(n=7)>$ controls } \\
\hline Right pons* & 12 & -25 & -37 & 116 & $>0.05$ & 0.001 & 3.5 \\
\hline \multicolumn{8}{|l|}{ Late assessment } \\
\hline \multicolumn{8}{|l|}{ Patients $(n=7)<$ controls } \\
\hline Right insula (BA47) & 46 & 14 & -2 & 610 & 0.001 & $<0.001$ & 5.2 \\
\hline Right cerebellum & 42 & -50 & -46 & 220 & 0.09 & $<0.001$ & 5.0 \\
\hline Right olfactory gyrus (BA25) & 4 & 13 & -16 & 110 & 0.09 & $<0.001$ & 4.8 \\
\hline
\end{tabular}

MNI coordinates are expressed in mm relative to the anterior commissure. Statistical maps were threshold for significance at $p<0.05$ corrected using the FWE (family-wise error) method with a cluster extent of 100 voxels. Results displayed with $p$ values $<0.05$ uncorrected are indicated with *. BA, Brodmann area; $M N I$, Montreal Neurological Institute space; $k_{E}$, cluster size in number of voxels

associated with morphological abnormalities except in patient \#7 previously described [6]. This patient with status epilepticus presented with T2-MRI hyperintensities in the right prefrontal cortex and caudate nucleus, possibly due to peri-ictal diffusion abnormalities. Arterial spin-labelling perfusion imaging revealed frontal hypoperfusion and cerebellar hyperperfusion in $2 / 7$ and $1 / 7$ patients, respectively (Table 1 ).

\section{Clinical and brain PET follow-up}

All patients clinically improved within 2 months of disease onset, with remaining symptoms of variable severity (Table 1). Follow-up at a median delay of 5.1 months after COVID-19 onset (range: $3.6-8.3$ ) confirmed that all patients, except one (\#4) had improved with the normal physical neurological examination and had recovered normal autonomy in daily living. However, they all still had an abnormal cognitive evaluation with at least an attention/executive deficit. They also presented with depression and anxiety of varying severity: two patients $(\# 2,7)$ had well-controlled symptoms under psychotropic treatments, and one patient (\#5) developed symptoms associated with post-traumatic stress disorder. After having partially recovered, patient \#4 subsequently relapsed with severe depression associated with psychotic symptoms, hallucinations, worsening cognitive impairment and extrapyramidal syndrome, 2.6 months after disease onset.

In agreement with the clinical course, brain metabolism improved significantly 1 month after the first examination (median delay 28 days, range: $21-56$ ) with less marked cortical hypometabolism and no more hypermetabolic areas 
Table 3 18F-FDG-PET findings in the whole group vs. healthy controls: results of ROI analysis in acute phase, one month later and 6 month after COVID-19 onset

\begin{tabular}{|c|c|c|c|c|}
\hline & \multicolumn{4}{|l|}{ SUV ratio } \\
\hline & Controls & Patients & & \\
\hline Anatomic location & & Baseline & Early assessment & Late assessment \\
\hline Right orbital frontal gyrus & $1.27 \pm 0.08$ & $1.03 \pm 0.10 \triangleleft(-19 \%)$ & $1.08 \pm 0.14 *(-15 \%)$ & $1.16 \pm 0.17(-9 \%)$ \\
\hline Left orbital frontal gyrus & $1.30 \pm 0.09$ & $1.09 \pm 0.12 \downarrow(-16 \%)$ & $1.16 \pm 0.12 *(-10 \%)$ & $1.25 \pm 0.15(-4 \%)$ \\
\hline Right middle frontal gyrus & $1.39 \pm 0.08$ & $1.10 \pm 0.11 \triangleleft(-20 \%)$ & $1.18 \pm 0.11 \downarrow(-15 \%)$ & $1.28 \pm 0.15(-8 \%)$ \\
\hline Left middle frontal & $1.35 \pm 0.09$ & $1.13 \pm 0.17 *(-16 \%)$ & $1.20 \pm 0.13 *(-11 \%)$ & $1.30 \pm 0.17(-4 \%)$ \\
\hline Right medial frontal gyrus & $1.25 \pm 0.08$ & $1.02 \pm 0.13 \downarrow(-19 \%)$ & $1.06 \pm 0.14 *(-15 \%)$ & $1.12 \pm 0.19(-10 \%)$ \\
\hline Left medial frontal gyrus & $1.24 \pm 0.08$ & $1.04 \pm 0.14 *(-16 \%)$ & $1.09 \pm 0.11 *(-12 \%)$ & $1.16 \pm 0.15(-6 \%)$ \\
\hline Right olfactory cortex & $1.07 \pm 0.07$ & $0.95 \pm 0.12 *(-12 \%)$ & $0.93 \pm 0.10 *(-13 \%)$ & $0.95 \pm 0.12 *(-11 \%)$ \\
\hline Left olfactory cortex & $1.07 \pm 0.08$ & $0.96 \pm 0.12 *(-10 \%)$ & $0.97 \pm 0.10 *(-10 \%)$ & $0.97 \pm 0.08 *(-10 \%)$ \\
\hline Right rectus gyrus & $1.34 \pm 0.08$ & $1.11 \pm 0.17 *(-18 \%)$ & $1.16 \pm 0.19 *(-14 \%)$ & $1.23 \pm 0.24(-9 \%)$ \\
\hline Left rectus gyrus & $1.32 \pm 0.08$ & $1.12 \pm 0.15 *(-15 \%)$ & $1.17 \pm 0.16^{*}(-11 \%)$ & $1.24 \pm 0.17(-6 \%)$ \\
\hline Anterior cingulate cortex & $1.34 \pm 0.11$ & $1.07 \pm 0.18 *(-20 \%)$ & $1.12 \pm 0.17 *(-17 \%)$ & $1.17 \pm 0.20 *(-13 \%)$ \\
\hline Posterior cingulate cortex & $1.64 \pm 0.13$ & $1.39 \pm 0.26 *(-15 \%)$ & $1.53 \pm 0.23(-7 \%)$ & $1.62 \pm 0.31(-1 \%)$ \\
\hline Right insula & $1.28 \pm 0.07$ & $1.11 \pm 0.09 \diamond(-14 \%)$ & $1.11 \pm 0.12 *(-14 \%)$ & $1.15 \pm 0.14 *(-10 \%)$ \\
\hline Left insula & $1.29 \pm 0.07$ & $1.11 \pm 0.08 \downarrow(-14 \%)$ & $1.14 \pm 0.10 *(-11 \%)$ & $1.20 \pm 0.13(-7 \%)$ \\
\hline Right inferior parietal cortex & $1.37 \pm 0.10$ & $1.13 \pm 0.17(-17 \%)$ & $1.25 \pm 0.17(-9 \%)$ & $1.37 \pm 0.23(0 \%)$ \\
\hline Left inferior parietal cortex & $1.31 \pm 0.09$ & $1.10 \pm 0.17(-15 \%)$ & $1.20 \pm 0.15(-8 \%)$ & $1.30 \pm 0.21(-1 \%)$ \\
\hline Early right occipital cortex & $1.34 \pm 0.08$ & $1.16 \pm 0.16(-10 \%)$ & $1.28 \pm 0.14(-5 \%)$ & $1.35 \pm 0.19(0 \%)$ \\
\hline Left occipital cortex & $1.30 \pm 0.09$ & $1.13 \pm 0.15(-11 \%)$ & $1.24 \pm 0.13(-5 \%)$ & $1.31 \pm 0.20(0 \%)$ \\
\hline Right middle temporal gyrus & $1.33 \pm 0.08$ & $1.15 \pm 0.10 \bullet(-13 \%)$ & $1.24 \pm 0.13(-6 \%)$ & $1.31 \pm 0.16(-1 \%)$ \\
\hline Left middle temporal gyrus & $1.28 \pm 0.08$ & $1.13 \pm 0.13 *(-11 \%)$ & $1.20 \pm 0.13(-6 \%)$ & $1.27 \pm 0.17(0 \%)$ \\
\hline Right inferior temporal gyrus & $1.25 \pm 0.07$ & $1.10 \pm 0.12 *(-12 \%)$ & $1.18 \pm 0.11(-6 \%)$ & $1.23 \pm 0.16(-2 \%)$ \\
\hline Left inferior temporal gyrus & $1.22 \pm 0.06$ & $1.08 \pm 0.14 *(-11 \%)$ & $1.15 \pm 0.13(-5 \%)$ & $1.20 \pm 0.16(-2 \%)$ \\
\hline Right amygdala & $0.96 \pm 0.06$ & $0.94 \pm 0.15(-2 \%)$ & $0.92 \pm 0.09(-3 \%)$ & $0.91 \pm 0.08(-5 \%)$ \\
\hline Left amygdala & $1.00 \pm 0.06$ & $0.97 \pm 0.14(-2 \%)$ & $0.96 \pm 0.11(-3 \%)$ & $0.96 \pm 0.09(-4 \%)$ \\
\hline Right hippocampus & $1.01 \pm 0.06$ & $0.96 \pm 0.18(-5 \%)$ & $0.94 \pm 0.15(-6 \%)$ & $0.95 \pm 0.15(-5 \%)$ \\
\hline Left hippocampus & $0.99 \pm 0.07$ & $0.96 \pm 0.17(-3 \%)$ & $0.95 \pm 0.14(-5 \%)$ & $0.95 \pm 0.14(-4 \%)$ \\
\hline Right parahippocampal gyrus & $1.05 \pm 0.05$ & $1.00 \pm 0.16(-5 \%)$ & $1.01 \pm 0.13(-3 \%)$ & $1.03 \pm 0.12(-1 \%)$ \\
\hline Left parahippocampal gyrus & $1.03 \pm 0.06$ & $0.97 \pm 0.15(-6 \%)$ & $0.99 \pm 0.11(-4 \%)$ & $1.00 \pm 0.10(-3 \%)$ \\
\hline Right caudate nucleus & $1.22 \pm 0.16$ & $0.81 \pm 0.24 \downarrow(-33 \%)$ & $0.87 \pm 0.24 *(-28 \%)$ & $0.96 \pm 0.24 *(-21 \%)$ \\
\hline Left caudate nucleus & $1.23 \pm 0.17$ & $0.91 \pm 0.23 *(-26 \%)$ & $1.00 \pm 0.26(-19 \%)$ & $1.07 \pm 0.25(-13 \%)$ \\
\hline Right putamen & $1.52 \pm 0.11$ & $1.37 \pm 0.20(-9 \%)$ & $1.43 \pm 0.23(-5 \%)$ & $1.47 \pm 0.22(-3 \%)$ \\
\hline Left putamen & $1.56 \pm 0.12$ & $1.43 \pm 0.17(-8 \%)$ & $1.47 \pm 0.25(-5 \%)$ & $1.51 \pm 0.24(-3 \%)$ \\
\hline Right thalamus & $1.36 \pm 0.12$ & $1.20 \pm 0.17(-12 \%)$ & $1.23 \pm 0.16(-10 \%)$ & $1.28 \pm 0.18(-6 \%)$ \\
\hline Left thalamus & $1.36 \pm 0.10$ & $1.25 \pm 0.15(-8 \%)$ & $1.26 \pm 0.14(-7 \%)$ & $1.30 \pm 0.18(-4 \%)$ \\
\hline Cerebellar hemispheres & $1.16 \pm 0.05$ & $1.03 \pm 0.11 *(-12 \%)$ & $1.04 \pm 0.06 \downarrow(-11 \%)$ & $1.06 \pm 0.08 *(-8 \%)$ \\
\hline Cerebellar vermis & $1.43 \pm 0.13$ & $1.43 \pm 0.20(0 \%)$ & $1.38 \pm 0.13(-3 \%)$ & $1.44 \pm 0.16(1 \%)$ \\
\hline
\end{tabular}

SUV ratio values expressed as mean \pm standard deviation and percent difference between patients and controls (value in brackets). Values with indicate a statistically significant difference between controls and patients with $p<0.005$ (bilateral Student's $t$ test). Values with $*$ indicate a statistically significant difference between controls and patients with $p<0.05$ (bilateral Student's $t$ test)

(Fig. 1). Six months after COVID-19 onset (median delay 5.9 months, range 4.9-6.5), brain metabolism measured on the whole patient group vs. controls, returned almost normal with mild residual hypometabolism in the rectus/olfactory gyrus (with $9 \%$ decrease vs. $-19 \%$ at baseline), right insula (10\% vs. $14 \%)$, anterior cingulate ( $-13 \mathrm{vs}-20 \%)$ and right caudate nucleus ( $-21 \%$ vs. $-33 \%$ ) (Fig. 1, Tables 2 and 3). However, when assessed individually, follow-up revealed some degrees 


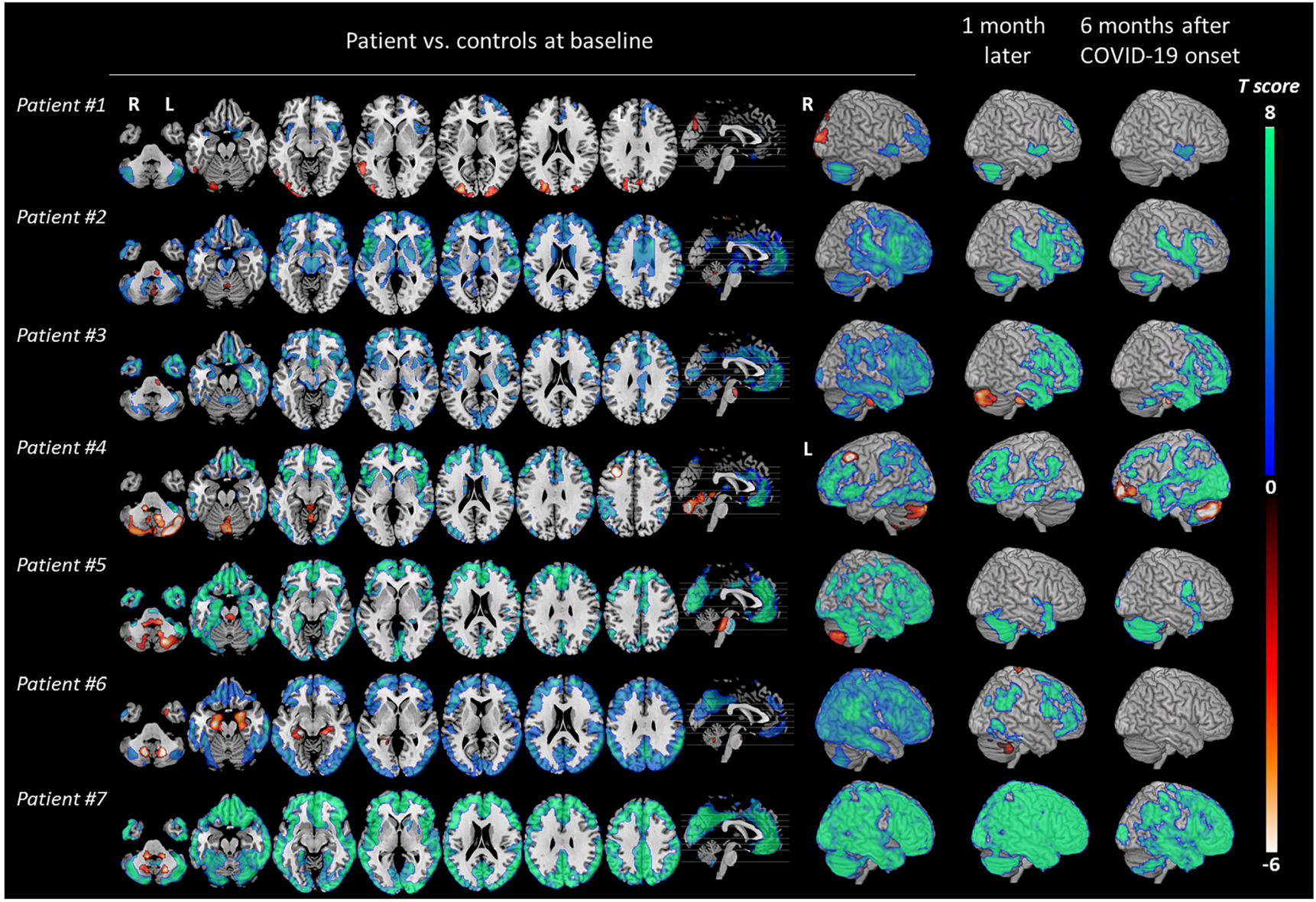

Fig. 2 Brain metabolism changes in each patient vs. controls $(n=32)$ in the acute phase, 1 month later and 6 months after COVID-19 onset. SPM T maps generated from patient \#1 to patient \#7 are displayed with a hot color scale for brain hypermetabolism $(p<0.05)$ and with a cool color scale for brain hypometabolism $(p<0.05$ corrected), in axial orthogonal views in neurological convention (right is right). R, right; L, left. 3D rendering shows for all patients the right hemisphere except in patient \#4 who presented hypometabolism in the left frontal cortex due to focal seizure of heterogeneity across patients. Metabolism returned to almost normal in three patients (\#1, 5, 6, Fig. 2, Table 4). In patients \#2, 3 and 7, the improvement was moderate with prefrontal hypometabolism persisting 6 months after disease onset. In agreement with clinical course, patient \#4 first improved and got worse again with severe hypometabolism appearing in the bilateral occipital cortex, and prefrontal and cerebellar hypermetabolisms (Fig. 2, Table 4).

\section{Discussion}

A pattern of 18F-FDG-PET/CT hypometabolism in a widespread cerebral network including the prefrontal cortex, anterior cingulate, insula and caudate nucleus was observed in seven patients with acute COVID-19-related encephalopathy. Longitudinal data suggest that this network remains mildly to severely impaired 6 months after disease onset.

Despite varied clinical symptomatology and variable degrees of cognitive impairment in acute COVID-19-related encephalopathy, a pattern of orbitofrontal, dorsolateral and mesiofrontal hypometabolism was found in all patients and was consistent with patients' prominent clinical frontal lobe syndrome. These results reinforce the hypothesis of major frontal lobe impairment in COVID-19-related encephalopathy, in line with [3-5]. These regions-of-interest and voxel-based 18F-FDGPET comparisons of a larger number of patients with healthy controls also provided further, unexpected insights, supporting the hypothesis of a large network dysfunction. Interestingly, every patient had severe hypometabolism within a network of functionally related areas, namely the insula, anterior cingulate and caudate nucleus. The insula has a key role in processing subjective awareness and integrating homeostatic information from the body. Insular disturbances could be implicated in the deregulation of respiratory failure perception which has been reported in patients with COVID-19 [7]. The anterior insula has a role in higher order consciousness and is highly connected with regions involved in social cognition, decision-making, motivational and affective processes, such as the orbitofrontal, prefrontal dorsolateral, anterior cingulate cortices, amygdala and hippocampus [8]. 
Table 4 SPM findings in each patient vs. healthy controls, in acute COVID-19-related encephalopathy

Location $(\mathrm{BA})$

Coordinates TAL $(\mathrm{mm})$

$x$

Case \#1 < controls

Right cerebellum

Right parahippocampal gyrus (BA53)

Right insula (BA47)

Left cerebellum

Left insula (BA13)

Right frontal orbital gyrus (BA10)

Case \#1 > controls

Left middle occipital gyrus (BA18)

Right cuneus (BA18)

Left middle temporal gyrus (BA37)

Left superior parietal (BA7)

Case \#2 < controls

Right inferior parietal (BA40)

Left cerebellum

Right precentral gyrus (BA6)

Right cerebellum

Left cerebellum

Case \#2 > controls

Right brainstem

Left precentral gyrus (BA1)

Right vermis*

Case \# $3<$ controls

Right olfactory gyrus (BA25)

Right primary visual cortex (BA17)

Left inferior parietal (BA39)

Right inferior parietal (BA40)

Case \#3 > controls

Left pons

$-6$

$-14$

$-28$

Case \#4 < controls

Right insula (BA47)

Left precuneus (BA7)

Left inferior temporal gyrus (BA21)

Left inferior occipital gyrus (BA19)

Case \#4 > controls

Left middle frontal gyrus (BA8)

Left cerebellum

Right cerebellum

Left vermis

64

$-22$

$-36$

$-12$

$-54$

$-12$

$-38$

$-70$

$10-28$

$-32$

$-10$

$-34$

70

$-42$

48

$-32$

24

$\begin{array}{llll}46 & -70 & -34 & 1937 \\ 20 & 6 & -20 & 373 \\ 46 & 16 & -6 & 750 \\ -42 & -62 & -44 & 1015 \\ -38 & 8 & -14 & 433 \\ 14 & 70 & -8 & 1360\end{array}$

Cluster size

$\mathrm{k}_{\mathrm{E}}$

Voxel level

$\begin{array}{llll}-28 & -88 & 10 & 1980 \\ 22 & -96 & 12 & 353 \\ -54 & -56 & -2 & 466 \\ -28 & -72 & 60 & 302\end{array}$

14

$-48$

$-36$

162

299

217

47128

350

417

1939

2031

$<0.001$

$<0.001$

$<0.001$

$<0.001$

$<0.001$

$<0.001$

$<0.001$

$<0.001$

$<0.001$

$<0.001$

$<0.001$

$<0.001$

0.001

$<0.001$

5.6

0.09

43896

2139

362

381

$<0.001$

$<0.001$

22.8

$<0.001$

$<0.001$

13.0

$<0.001$

$<0.001$

11.5

0.001

$<0.001$

11.1

$\begin{array}{rrr}48 & 16 & -4 \\ -6 & -66 & 52 \\ -62 & -28 & -18 \\ -40 & -74 & -10\end{array}$

378

0.006

0.001

10.5

25911
2811
802

$<0.001$

$<0.001$

$<0.001$

$<0.001$

$<0.001$

$<0.001$

$<0.001$

9.3

18.4

10.8

10.4

$<0.001$

$<0.001$

$<0.001$

38.1

44

565

$<0.001$

$<0.001$

18.3

5942

$<0.001$

$<0.001$ 
Table 4 (continued)

\begin{tabular}{|c|c|c|c|c|c|c|c|}
\hline \multirow[t]{2}{*}{ Location (BA) } & \multicolumn{3}{|c|}{ Coordinates TAL (mm) } & \multirow{2}{*}{$\begin{array}{l}\text { Cluster size } \\
\mathrm{k}_{\mathrm{E}}\end{array}$} & \multicolumn{3}{|c|}{ Voxel level } \\
\hline & $x$ & $y$ & $z$ & & P corr & P uncorr & $T$ score \\
\hline \multicolumn{8}{|l|}{ Case $\# 5<$ controls } \\
\hline Right cerebellum & 44 & -48 & -50 & 46901 & $<0.001$ & $<0.001$ & 22.3 \\
\hline Right superior temporal gyrus (BA41) & 66 & -24 & 14 & 473 & $<0.001$ & $<0.001$ & 14.9 \\
\hline Right middle occipital gyrus (BA39) & 36 & -78 & 32 & 410 & $<0.001$ & $<0.001$ & 9.7 \\
\hline \multicolumn{8}{|l|}{ Case $\# 5<$ controls } \\
\hline Right pons & 10 & -34 & -34 & 669 & $<0.001$ & $<0.001$ & 11.1 \\
\hline Right cerebellum & 22 & -68 & -42 & 1261 & $<0.001$ & $<0.001$ & 10.8 \\
\hline Left cerebellum & -14 & -66 & -44 & 411 & 0.003 & $<0.001$ & 7.1 \\
\hline Left cerebellum & -36 & -60 & -50 & 153 & 0.02 & $<0.001$ & 6.4 \\
\hline \multicolumn{8}{|l|}{ Case\# $6<$ controls } \\
\hline Right inferior temporal (BA20) & 64 & -44 & 26 & 79991 & $<0.001$ & $<0.001$ & 24.1 \\
\hline Right cerebellum & 40 & -70 & -52 & 1054 & $<0.001$ & $<0.001$ & 13.1 \\
\hline Left cerebellum & -34 & -74 & -52 & 1015 & 0.001 & $<0.001$ & 11.1 \\
\hline \multicolumn{8}{|l|}{ Case \#6 > controls } \\
\hline Left cerebellum & -12 & -64 & -38 & 558 & $<0.001$ & $<0.001$ & 17.8 \\
\hline Right hippocampus (BA54) & -22 & -12 & -24 & 1157 & $<0.001$ & $<0.001$ & 16.6 \\
\hline Right hippocampus (BA54) & 20 & -8 & -16 & 1154 & $<0.001$ & $<0.001$ & 14.1 \\
\hline \multicolumn{8}{|l|}{ Case $\# 7<$ controls } \\
\hline Right cuneus (BA18) & 18 & -98 & 8 & 106715 & $<0.001$ & $<0.001$ & 24.0 \\
\hline \multicolumn{8}{|l|}{ Case \#7 > controls } \\
\hline Right cerebellum & 22 & -64 & -42 & 223 & $<0.001$ & $<0.001$ & 10.9 \\
\hline Left pons & -12 & -42 & -40 & 389 & $<0.001$ & $<0.001$ & 9.5 \\
\hline Right pons & 14 & -40 & -40 & 144 & $<0.001$ & $<0.001$ & 8.6 \\
\hline
\end{tabular}

MNI coordinates are expressed in mm relative to the anterior commissure. Statistical maps were threshold for significance at $p<0.05$ corrected using the FWE (family-wise error) method with a cluster extent of 100 voxels. Results displayed with $p$ values $<0.05$ uncorrected are indicated with *. BA, Brodmann area; $M N I$, Montreal Neurological Institute space; $k_{E}$, cluster size in number of voxels

The anterior insula is also connected with the caudate nucleus which is involved in many associative, executive, motivational and affective processes. Moreover, in our series, the cerebellum was concerned by metabolism modifications, while this structure is implicated in the modulation of cognition (as executive function, attention, language) and, in affects via functional connexions as limbic structures or fronto-parietal cortex [9]. Thus, the hypometabolism observed in this network could be the neural substrate of motivational and emotional disturbances observed in our patients. In line with [4], our findings also suggest an impairment of the thalamus and pons, which could contribute to respiratory control impairment in some patients in the acute phase.

Clinical follow-up with longitudinal PET studies in COVID-19-related encephalopathy are still lacking in the literature. In our series, clinical, neurological examination and brain metabolism improved early in the course of the disease. However, attention/executive deficit with prefrontal hypometabolism was persisting in all patients 6 months after COVD-19 onset. Lasting anxio-depressive symptoms were also observed in agreement with persistent hypometabolism in the right insula which is involved in post-traumatic stress disorder [10]. Our data also reveals heterogeneity in patient outcomes. While some patients almost returned to normal metabolism (even those with initial widespread decrease), others partially improved or worsened. After having first improved, patient \#4 subsequently worsened with clinical symptoms and a new pattern of brain hypometabolism which raises the question of an associated neurodegenerative disorder.

Underlying mechanisms of COVID-19-related encephalopathy are still debated. Our findings, such as the increased IL-6 levels in the blood and CSF in some patients 
may support a para-infectious cytokine release, postinfectious antibody- or cell-mediated immune mechanism. Cytokines can pass the blood-brain barrier, induce central inflammatory responses and influence neurotransmitter metabolism and neural plasticity. They can induce dysfunction in areas implicated in emotional and behavioural regulation and cognition (such as the prefrontal cortex, basal ganglia) and fear and anxiety-related regions (such as the amygdala, insula and anterior cingulate cortex) [11]. The mesiotemporal and/or cerebellar hypermetabolism in the acute phase, disappearing during the course of the disease with clinical recovery, also supports an immune mechanism [12]. No patient had SARSCoV-2 in the CSF and/or meningitis nor 18F-FDG-PET anomalies limited to the olfactory gyrus that could corroborate a direct viral neuro-invasion.

Considering that $18 \mathrm{~F}-\mathrm{FDG}-\mathrm{PET}$ findings are more strongly associated with clinical symptoms, disease course and status than MRI (in the exception of cerebrovascular events) [12, 13], it should be considered for the initial workup and for monitoring treatment in COVID-related encephalopathy. It could also help to further decipher the neuronal substrates of COVID-19-associated encephalopathy. Further longitudinal 18F-FDG-PET/CT studies with a larger sample size are needed to understand the pathophysiological bases of metabolism changes.

Acknowledgements The authors thank the Cohort COVID-19 Neurosciences (CoCo Neurosciences), study sponsored by APHP- ICM and consequently the CoCo-Neurosciences study group and the COVID SMIT PSL study group. Steering Committee (Pitié-Salpêtrière Hospital, Paris): Cecile Delorme, Jean-Christophe Corvol, Jean-Yves Delattre, Stephanie Carvalho, Sandrine Sagnes. Scientific Committee (PitiéSalpêtrière Hospital, Paris): Bruno Dubois, Vincent Navarro, Celine Louapre, Tanya Stojkovic, Ahmed Idbaih, Charlotte Rosso, David Grabli, Ana Zenovia Gales, Bruno Millet, Benjamin Rohaut, Eleonore Bayen, Sophie Dupont, Gaelle Bruneteau, Stephane Lehericy, Danielle Seilhean, Alexandra Durr, Aurelie Kas, Foudil Lamari, Marion Houot, Vanessa Batista Brochard. Principal investigators: Pitié-Salpêtrière Hospital (Paris): Sophie Dupont, Catherine Lubetzki, Danielle Seilhean, Pascale Pradat-Diehl, Charlotte Rosso, Khe Hoang-Xuan, Bertrand Fontaine, Lionel Naccache, Philippe Fossati, Isabelle Arnulf, Alexandra Durr, Alexandre Carpentier, Stephane Lehericy, Yves Edel; Foch Hospital (Suresnes): Anna Luisa Di Stefano; Rothschild Hospital (Paris): Gilberte Robain, Philippe Thoumie; Avicenne Hospital (Bobigny): Bertrand Degos; Sainte-Anne Hospital (Paris): Tarek Sharshar; Saint-Antoine Hospital (Paris): Sonia Alamowitch, Emmanuelle Apartis-Bourdieu, Charles-Siegried Peretti; Saint-Louis Hospital (Paris): Renata Ursu; Tenon Hospital (Paris): Nathalie Dzierzynski; Charles Foix Hospital (Ivry): Kiyoka Kinugawa Bourron, Joel Belmin, Bruno Oquendo, Eric Pautas, Marc Verny. Co-investigators: Pitié-Salpêtrière Hospital (Paris): Cecile Delorme, Jean-Christophe Corvol, Jean-Yves Delattre, Yves Samson, Sara Leder, Anne Leger, Sandrine Deltour, Flore Baronnet, Ana Zenovia Gales,Stephanie Bombois, Mehdi Touat, Ahmed Idbaih, Marc Sanson, Caroline Dehais, Caroline Houillier, Florence Laigle-Donadey, Dimitri Psimaras, Agusti
Alenton, Nadia Younan, Nicolas Villain, David Grabli, Maria del Mar Amador, Gaelle Bruneteau, Celine Louapre, Louise-Laure Mariani, Nicolas Mezouar, Graziella Mangone, Aurelie Meneret, Andreas Hartmann, Clement Tarrano, David Bendetowicz, Pierre-François Pradat, Michel Baulac, Sara Sambin, François Salachas, Nadine Le Forestier, Phintip Pichit, Florence Chochon, Adele Hesters, Bastien HerlinAn Hung Nguyen, Valerie Procher, Alexandre Demoule, Elise Morawiec, Julien Mayaux, Morgan Faure, Claire Ewenczyk, Giulia Coarelli, Anna Heinzmann, Perrine Charles, Tanya Stojkovic, Marion Masingue, Guillaume Bassez, Vincent Navarro, Isabelle An, Yulia Worbe, Virginie Lambrecq, Rabab Debs, Esteban Munoz Musat, Timothee Lenglet, Virginie Lambrecq, Aurelie Hanin, Lydia Chougar, Nathalia Shor, Nadya Pyatigorskaya, Damien Galanaud, Delphine Leclercq, Sophie Demeret, Benjamin Rohaut, Albert Cao, Clemence Marois, Nicolas Weiss, Salimata Gassama, Loic Le Guennec, Vincent Degos, Alice Jacquens, Thomas Similowski, Capucine Morelot-Panzini, Jean-Yves Rotge, Bertrand Saudreau, Bruno Millet, Victor Pitron, Nassim Sarni, Nathalie Girault, Redwan Maatoug, Ana Zenovia Gales, Smaranda Leu, Eleonore Bayen, Lionel Thivard, Karima Mokhtari, Isabelle Plu; Sainte-Anne Hospital (Paris): Bruno Gonçalves; SaintAntoine Hospital (Paris): Laure Bottin, Marion Yger; Rothschild Hospital (Paris): Gaelle Ouvrard, Rebecca Haddad; Charles Foix Hospital (Ivry): Flora Ketz, Carmelo Lafuente, Christel Oasi. Other Contributors: Associated centres (Lariboisière Hospital, Paris): Bruno Megabarne, Dominique Herve; Clinical Research Associates (ICM, Pitié-Salpêtrière Hospital, Paris): Haysam Salman, Armelle RamettiLacroux, Alize Chalançon, Anais Herve, Hugo Royer, Florence Beauzor, Valentine Maheo, Christelle Laganot, Camille Minelli, Aurelie Fekete, Abel Grine, Marie Biet, Rania Hilab, Aurore Besnard, Meriem Bouguerra, Gwen Goudard, Saida Houairi, Saba Al-Youssef, Christine Pires, Anissa Oukhedouma, Katarzyna Siuda-Krzywicka, Tal Seidel Malkinson; (Saint-Louis Hospital, Paris): Hanane Agguini; (Foch Hospital, Suresnes): Hassen Douzane; Data Manager (ICM, Paris): Safia Said; Statistician (ICM, Paris): Marion Houot.

Authors' contributions All authors contributed to the study conception and design. Material preparation, data collection and analysis were performed by Aurélie Kas, Marine Soret, Nadya Pyatigoskaya, Marie-Odile Habert, Adèle Hesters, Loic Le Guennec, Olivier Paccoud, Stéphanie Bombois and Cécile Delorme. The first draft of the manuscript was written by Aurélie Kas and all authors commented on previous versions of the manuscript. All authors read and approved the final manuscript.

Data Availability Not publicly available.

\section{Compliance with ethical standards}

Conflict of interest The authors declare that they have no conflict of interest.

Ethics approval The study was approved by the Sorbonne University Ethics Committee (CER-202028 on 24/04/2020).

Consent to participate Patients received information and agreed to the use of their medical data in accordance with French regulations.

Consent for publication Patients received information and agreed to the use of their medical data in accordance with French regulations.

Code availability Not applicable 


\section{References}

1. Ellul MA, Benjamin L, Singh B, Lant S, Michael BD, Easton A, et al. Neurological associations of COVID-19. Lancet Neurol. 2020;19:767-83.

2. Chougar L, Shor N, Weiss N, Galanaud D, Leclercq D, Mathon B, et al. Retrospective observational study of brain magnetic resonance imaging findings in patients with acute SARS-CoV-2 infection and neurological manifestations. Radiology. 2020. https://doi. org/10.1148/radiol.2020202422.

3. Cani I, Barone V, D'Angelo R, Pisani L, Allegri V, Spinardi L, et al. Frontal encephalopathy related to hyperinflammation in COVID-19. J Neurol. 2020; http://link.springer.com/10.1007/ s00415-020-10057-5. Accessed 11 Sept 2020.

4. Guedj E, Million M, Dudouet P, Tissot-Dupont H, Bregeon F, Cammilleri S, et al. 18F-FDG brain PET hypometabolism in postSARS-CoV-2 infection: substrate for persistent/delayed disorders? Eur J Nucl Med Mol Imaging. 2020. https://doi.org/10.1007/ s00259-020-04973-X.

5. Delorme C, Paccoud O, Kas A, Hesters A, Bombois S, Shambrook $\mathrm{P}$, et al. Covid-19-related encephalopathy: a case series with brain FDG-PET/CT findings. Eur J Neurol. 2020; https://www.ncbi.nlm. nih.gov/pmc/articles/PMC7461074. Accessed 5 Sept 2020.

6. Le Guennec L, Devianne J, Jalin L, Cao A, Galanaud D, Navarro V, et al. Orbitofrontal involvement in a neuroCOVID-19 patient.
Epilepsia. 2020; https://onlinelibrary.wiley.com/doi/abs/10.1111/ epi.16612. Accessed 6 Sept 2020.

7. Nouri-Vaskeh M, Sharifi A, Khalili N, Zand R, Sharifi A. Dyspneic and non-dyspneic (silent) hypoxemia in COVID-19: possible neurological mechanism. Clin Neurol Neurosurg. 2020;106217.

8. Christopher L, Koshimori Y, Lang AE, Criaud M, Strafella AP. Uncovering the role of the insula in non-motor symptoms of Parkinson's disease. Brain. 2014;137:2143-54.

9. Schmahmann JD. The cerebellum and cognition. Neurosci Lett. 2019. https://doi.org/10.1016/j.neulet.2018.07.005.

10. Jeong H, Chung YA, Ma J, et al. Diverging roles of the anterior insula in trauma-exposed individuals vulnerable or resilient to posttraumatic stress disorder. Sci Rep. 2019. https://doi.org/10.1038/ s41598-019-51727-3.

11. Felger JC. Imaging the role of inflammation in mood and anxietyrelated disorders. Curr Neuropharmacol. 2018;16:533-58.

12. Morbelli S, Djekidel M, Hesse S, Pagani M, Barthel H. Role of 18F-FDG-PET imaging in the diagnosis of autoimmune encephalitis. Lancet Neurol. 2016;15:1009-10.

13. Morbelli S, Ekmekcioglu O, Barthel H, Albert NL, Boellaard R, Cecchin D, et al. COVID-19 and the brain: impact on nuclear medicine in neurology. Eur J Nucl Med Mol Imaging. 2020. https://doi. org/10.1007/s00259-020-04965-X.

Publisher's note Springer Nature remains neutral with regard to jurisdictional claims in published maps and institutional affiliations. 